\title{
Caracteristique Et Vitesse De Propagation D'une Onde De Maree Semi-Diurne A Inegalite Diurne Au Port Autonome D'abidjan : Incidence De La Morphologie Du Systeme Lagunaire Ebrie
}

\author{
Samassy Rokyatou \\ Akobe Apie Colette \\ Université Felix Houphouët Boigny / UFR STRM \\ Fossi Yannick Fosti \\ Univerité de Douala / Faculté des sciences \\ Monde Sylvain \\ Université Felix Houphouët Boigny / UFR STRM \\ Angora Aman \\ Université Felix Houphouët Boigny / UFR SSMT \\ Sangare Seydou
}

Port Autonome d'Abidjan / Service Hydrographie et Océanographie

Doi:10.19044/esj.2018.v14n26p282 URL:http://dx.doi.org/10.19044/esj.2018.v14n26p282

\begin{abstract}
The autonomous port of Abidjan, qualified as "lung of the Ivorian economy", is ranked first in West Africa and second in Africa, after the port of Durban in South Africa.

It is a transhipment and container port, thanks to the Vridi channel with a depth of $15 \mathrm{~m}$, the boats with deep draft can dock in deep water. Thus for the safety of the port navigation, it is important to know the speed of propagation of the tidal wave in order to know exactly the tide times in all parts of the Ebrié lagoon. This lagoon in connection with inflows of external waters considerably modify its hydrodynamism. For a mastery of the hydrodynamics of this complex environment we have subdivided the lagoon into three parts depending on the different inputs (fluvial / marine) or not. As a result, the tidal wave spreads much faster in the western part with a speed of $0.83 \mathrm{~ms}^{-1}$ over 1 $\mathrm{km}$, than in the eastern part of the Ebrié Lagoon, is a speed of $0.38 \mathrm{~m} \cdot \mathrm{s}^{-1}$. It is also slowing down in the Vridi Channel $0.79 \mathrm{~m} . \mathrm{s}^{-1}$. Thus the propagation of the tidal wave in the lagoon would be hampered by fluvial inputs and also by a restricted basin such as the Vridi Canal. In addition, the marine influence is dominant in the dry season, however in high flood season the tide phenomenon
\end{abstract}


is more important in the lagoon. The ebb tide is faster and longer than the lagoon flow, but the reverse phenomenon is observed at sea.

Keywords: Spread, tide, speed, port, Abidjan

\section{Resume}

Le port autonome d'Abidjan, qualifié de « poumon de l'économie ivoirienne », est classé au premier rang de l'Afrique de l'Ouest et au deuxième en Afrique, après le port de Durban en Afrique du Sud. C'est un port en transbordement et à conteneurs, grâce au canal de Vridi d'une profondeur de $15 \mathrm{~m}$, les bateaux à grand tirant d'eau peuvent accoster en eau profonde. Ainsi pour la sécurité de la navigation portuaire, il est important de connaitre la vitesse de propagation de l'onde de marée afin de savoir avec exactitudes les heures de marée dans toutes les parties de la lagune Ebrié. Pour une maitrise de l'hydrodynamisme de ce milieu complexe nous avons subdivisé la lagune en trois parties en fonction des différents apports (fluviales/marines). L'application de la méthode de Mahan (2009) aux données de 15 marégraphes installés dans presque toute la lagune Ebrié. Montre que l'onde de marée se propage beaucoup plus vite dans la partie Ouest avec une vitesse de 0,83 m.s ${ }^{1}$ sur $1 \mathrm{~km}$, que dans la partie Est de la lagune Ebrié soit une vitesse de 0,38 $\mathrm{m} . \mathrm{s}^{-1}$. Elle est également ralentit dans le Canal de Vridi $\left(0,79 \mathrm{~m} . \mathrm{s}^{-1}\right)$. Ainsi, la propagation de l'onde de marée en lagune serait entravée par les apports fluviaux et aussi par un bassin restreint tel le Canal de Vridi. En outre, l'influence marine est dominante en saison sèche, tandis qu'en saison de grande crue, le phénomène de marée est plus important en lagune. Le jusant est plus rapide et long que le flot en lagune, par contre le phénomène inverse est observé en mer.

Mots-clés : Propagation, marée, vitesse, port, Abidjan

\section{INTRODUCTION}

Autrefois La lagune Ebrié était uniquement ouvert sur la mer par l'embouchure temporaire du fleuve Comoé à Grand-Bassam. Depuis 1950, la lagune est en contact permanent avec la mer par le canal artificiel de Vridi (ouvrage de $2700 \mathrm{~m}$ de long, de $300 \mathrm{~m}$ de large et profond d'une vingtaine de mètres). Dans ce travail, nous nous intéresserons seulement à cette seconde ouverture.

Relié au golfe de Guinée par le canal de Vridi et avec une surface de $566 \mathrm{~km}^{2}$, le système lagunaire Ebrié est le plus étendu d'Afrique de l'Ouest. Il s'étire sur $125 \mathrm{~km}$ le long du littoral-ivoirien entre $3^{\circ} 40^{\prime}$ et $4^{\circ} 50^{\prime} \mathrm{W}$, à la latitude de $5^{\circ} 20 \mathrm{~N}$ (Varlet, 1978). Cette lagune en rapport avec des apports 
d'eaux externes modifient considérablement son hydrodynamisme (Ramany, 1979 ; Monde, 2004 ; Wango, 2009).

Le canal de Vridi donne l'accès aux différents navires au Port Autonome d'Abidjan qui est le principal poumon de l'économie ivoirienne. Dans ce canal, la marée se propage lentement avec de haute amplitude par rapport aux autres parties de la lagune (Samassy et al, 2017).

Dans l'optique de pérenniser la dynamique du Port d'Abidjan, il serait intéressant de comprendre les différents facteurs qui influent sur la marée dans ce canal.

Pour ce faire, une analyse des caractéristiques et de la vitesse de propagation de l'onde de marée dans les différents secteurs du système lagunaire Ebrié sera faite. Pour la réalisation de cette étude, dans ce milieu à morphologie complexe, nous avons subdivisé la lagune Ebrié en trois parties (le chenal Est, Ouest et l'Estuaire) à partir d'une ancienne classification établit par Varlet (1978).

\section{APPROCHE METHODOLOGIQUE}

Les données utilisées pour la réalisation de cette étude sont d'une part des enregistrements de l'année 1979 de plusieurs stations marégraphiques. Validées et effectuées par des marégraphes à flotteur de type OTT du service hydrographie du Port Autonome d'Abidjan et d'autre part des données de 1954 validées par Varlet (1958).

Ces données ont été nunérisées puis digitalisées par le logiciel "Surfur" avant son traitement par le logiciel T-tide. Elles sont issues de quinze marégraphes dispersés en lagune Ebrié et en mer. Dans la lagune Ebrié, les marégraphes sont reparties dans les trois (3) unités morphodynamiques :

- la première partie représente "l'estuaire", elle se compose d'un bassin central, orienté est-ouest, large de 1 à $7 \mathrm{~km}$, comportant le Canal de Vridi large de $370 \mathrm{~m}$ et profond d'une vingtaine de mètres, reliant la lagune Ebrié à l'Océan Atlantique (Dufour, 1982).

Les marégraphiques I.1 et I.2 se trouvent dans l'océan Atlantique, I.3 à la sortie du Canal de Vridi en lagune. I.4 et I.5 sont respectivement à petit Bassam et vers l'Ile Boulay.

- La seconde partie représente le "chenal Est" avec le fleuve Comoé à son extrémité. Dans cette zone, la lagune Ebrié rejoint le Comoé à Moossou avec les marégraphes II.1 et II.2 situés vers l'Ile des chauves-souris. II.3 se trouve vers l'Ile Vitré, II.4 à Moossou et II.5 en lagune Ouladine.

- La troisième partie représente le "chenal Ouest", bordé par le Canal d'Asagny et sans influence fluviale. Elle comporte les marégraphes III.1, III.2, III.3, III.4 et III.5 situés respectivement à 1'Ile 58, à l'Ile Cornuet, l'ile aux pigeons, à Potou 31 et à Asagny. (figure1). 
La marée a été caractérisée par quelques grandeurs, liées aux composantes harmoniques de la marée tels que : l'établissement moyen, l'âge de la marée et l'asymétrie tidale (Gouriou, 2012). La vitesse de propagation quant à elle a été déterminée selon la méthode utilisée par Mahan et al, (2009) pour le port de San-Pedro. Ainsi les phases relatives de chaque composante harmonique de la marée par rapport au fuseau horaire GMT permettent entre autres l'estimation du temps de propagation de l'onde de marée et sa vitesse entre deux points du globe. La composante harmonique semi-diurne (M2) est la composante de la marée la plus importante à chaque station marégraphique. Ainsi, Temps $(h)=($ phase 1 -Phase 2$) *$ (période de $M 2 / 360)$, où phase 1 et phase 2 représentent les phases respectives de l'onde M2 qui a une période de 12,42 heures. 


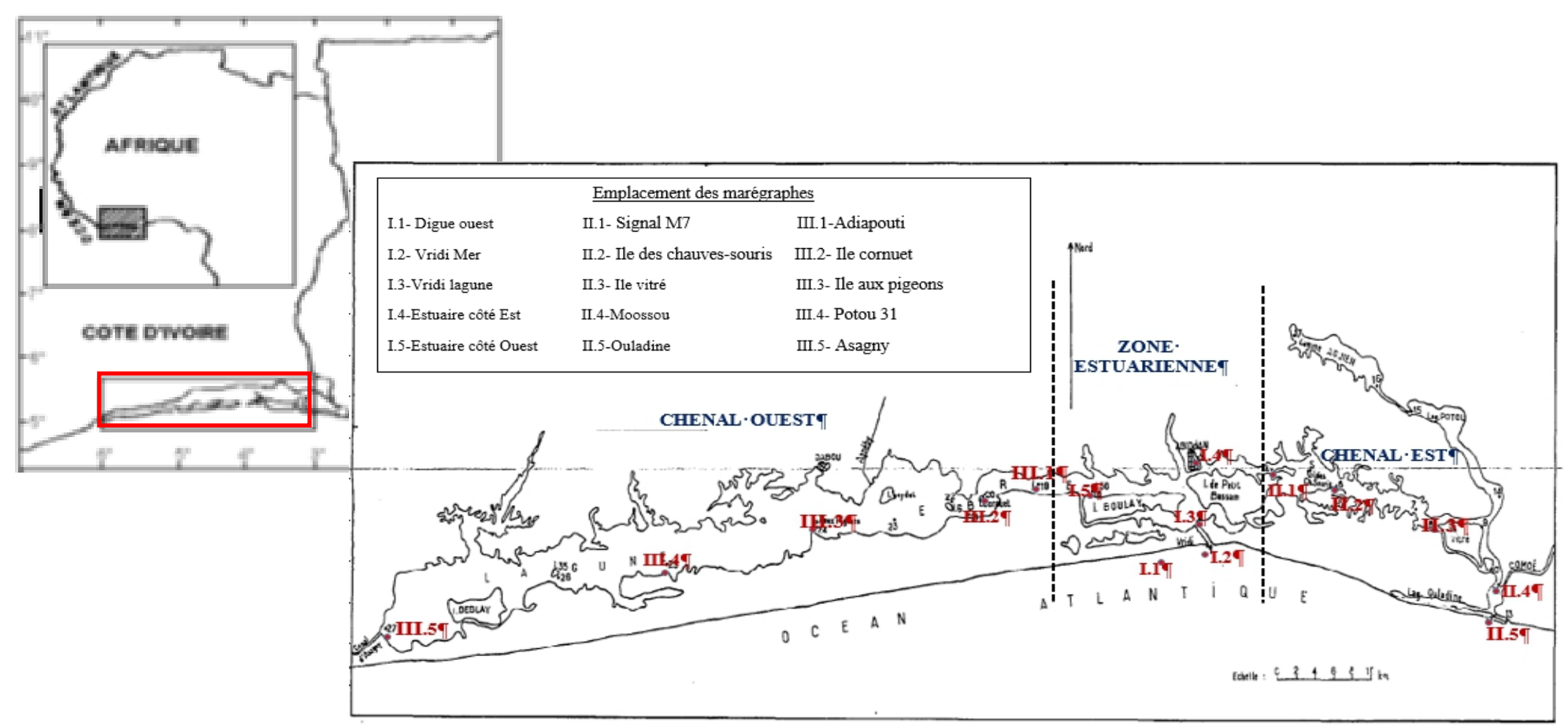

Figure $1:$ stations marégraphiques réparties en lagune Ebrié et en mer 


\section{RESULTATS}

\section{Oscillation annuelle de la marée dans la lagune Ebrié}

Durant un cycle annuel, le signal de marée présente la même allure dans le Canal de Vridi et en lagune. On observe une oscillation sinusoïdale marquée par des pics. Et les espaces entre les figures représentent des données manquantes au cours de l'enregistrement.

Sur la figure 2, on observe en lagune deux pics : le premier pic de l'année avec une hauteur de 1,47 m est observé le 11 juin 1979 (période correspondant à la grande saison pluvieuse) et le second avec une hauteur de 1,7 m, est observé le 24 octobre 1979 (grande saison de crue).

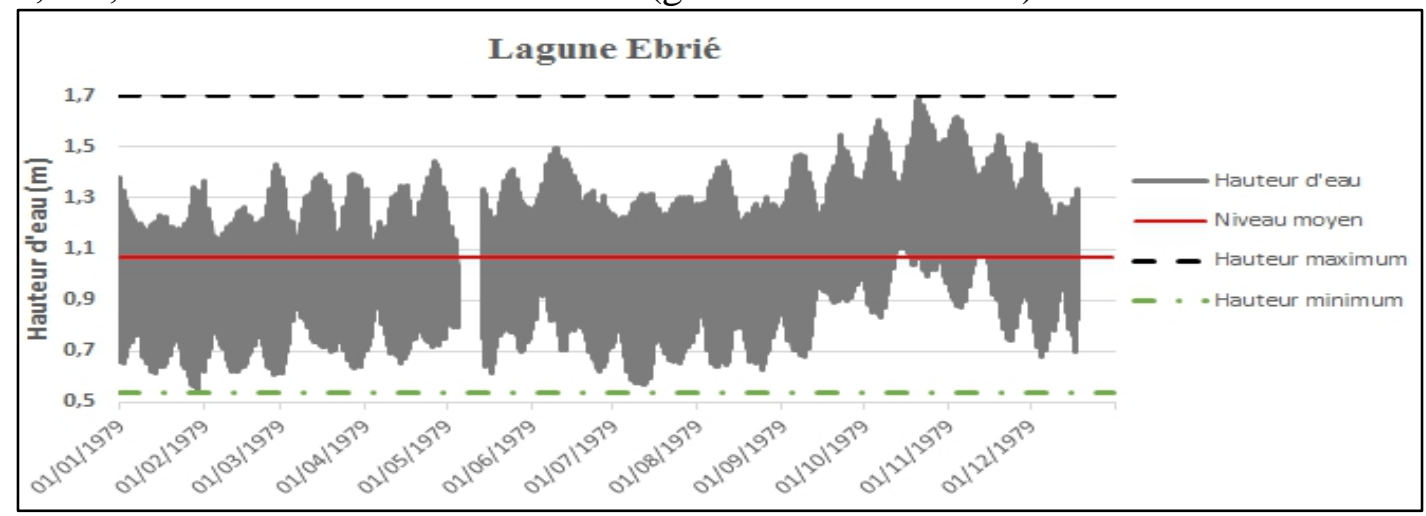

Figure 2: Différents niveaux d'eaux observés en lagune durant un cycle annuel

Dans le Canal de Vridi trois pics sont observés durant l'année (figure 3) : le premier avec une hauteur d'eau de 1,61 m est observé le 26 avril (grande saison marine). Le second est observé le 8 aout (période d'entrée importante d'eau marine) avec un niveau d'eau de 1,64 $\mathrm{m}$. Le troisième avec une hauteur de 1,81 m est observée le 4 et 22 octobre (période de grande crue).

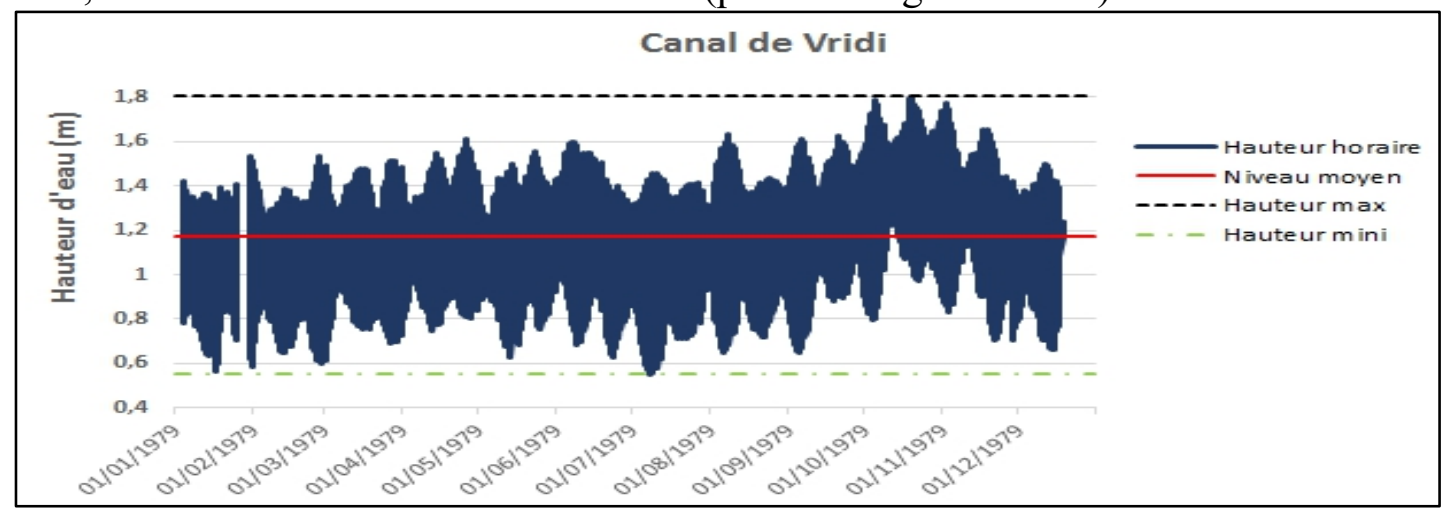

Figure 3 : Différents niveaux d'eaux observés dans le Canal de Vridi durant un cycle annuel 
Par contre en mer contrairement aux deux premiers sites, le signal de marée présente une allure presque linéaire. Ce qui montre que les niveaux d'eau en mer ne sont ni influencer par la crue du fleuve Comoé, ni par la saison pluvieuse. Par contre les plus hauts niveaux d'eaux ont été observés le 14 avril 1979 (période de grande saison marine) avec une hauteur de 1,54 m (figure 4).

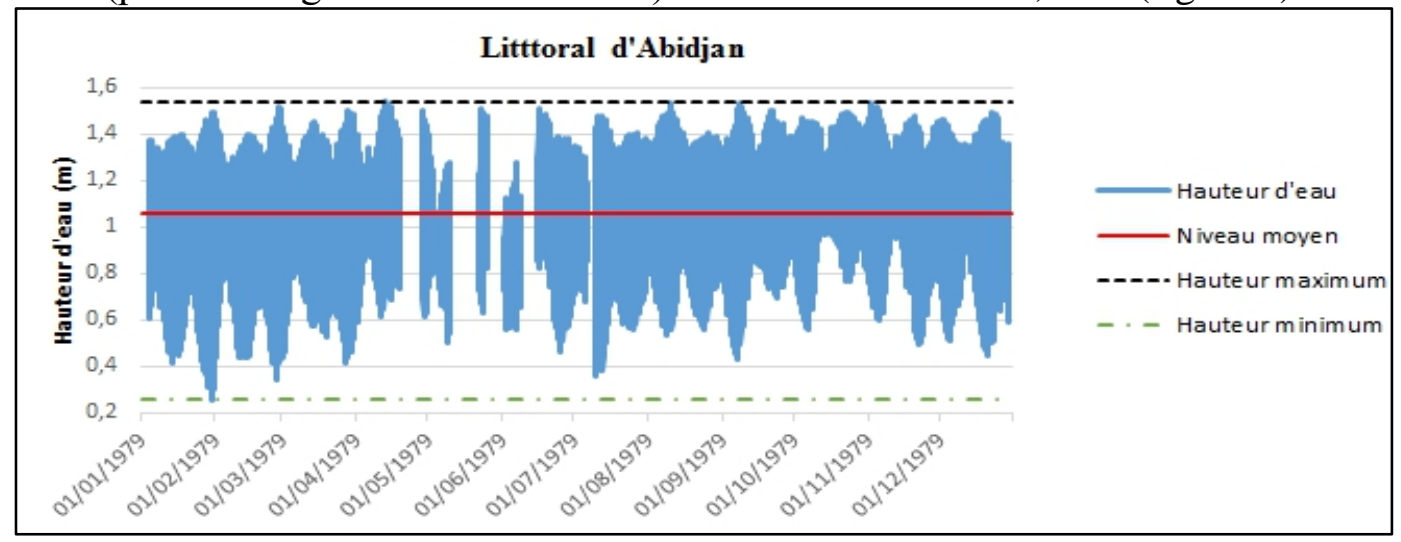

Figure 4: Différents niveaux d'eaux observés en mer durant un cycle annuel

\section{Caractérisation de l'onde de marée dans le littoral de côte d'ivoire}

Les principales composantes harmoniques du littoral ivoirien sont $\mathrm{K} 1$, $\mathrm{O} 1, \mathrm{P} 1$ et $\mathrm{Q} 1$ pour les ondes diurnes, et $\mathrm{M} 2, \mathrm{~S} 2, \mathrm{~K} 2$ et $\mathrm{N} 2$ pour les ondes semidiurnes. Elles contiennent à elles seules l'essentiel de l'énergie du signal de marée et sont parfois les seules prises en compte pour des études de première approximation (Simon, 2007). Les fréquences les plus élevées sur le littoral ivoirien sont celles des composantes semi-diurnes, ensuite les composantes diurnes, puis les longues périodes et enfin les quart-diurnes (tableau I).

Tableau I : Principales composantes harmoniques en mer

\begin{tabular}{llll}
\hline Onde & Fréquence & Amplitude $(\mathbf{m})$ & Phase \\
\hline *M2 & 0,0805114 & 0,2759 & 124,96 \\
*S2 & 0,0833333 & 0,1157 & 155,3 \\
*N2 & 0,0789992 & 0,0619 & 114,66 \\
*K2 & 0,0835615 & 0,0315 & 177,7 \\
*K1 & 0,0417807 & 0,0852 & 354,59 \\
*O1 & 0,0387307 & 0,0319 & 322,91 \\
*P1 & 0,0415526 & 0,0267 & 337,91 \\
*SSA & 0,0002282 & 0,0527 & 59,55 \\
*M4 & 0,1610228 & 0,0129 & 57,08 \\
\hline
\end{tabular}

L'amplitude de l'onde M2 avec 0,2759 m et une phase de $124,96^{\circ}$ est la plus élevée des ondes semi-diurnes, ainsi que celle de toutes les autres ondes. L'amplitude de $\mathrm{K} 1$ avec 0,0852 m est au-dessus des ondes diurnes. Les 
ondes longues périodes donnent une amplitude de $0,0527 \mathrm{~m}$ et les quartdiurnes présentent une très faible amplitude de $0,0129 \mathrm{~m}$ (figure 5).

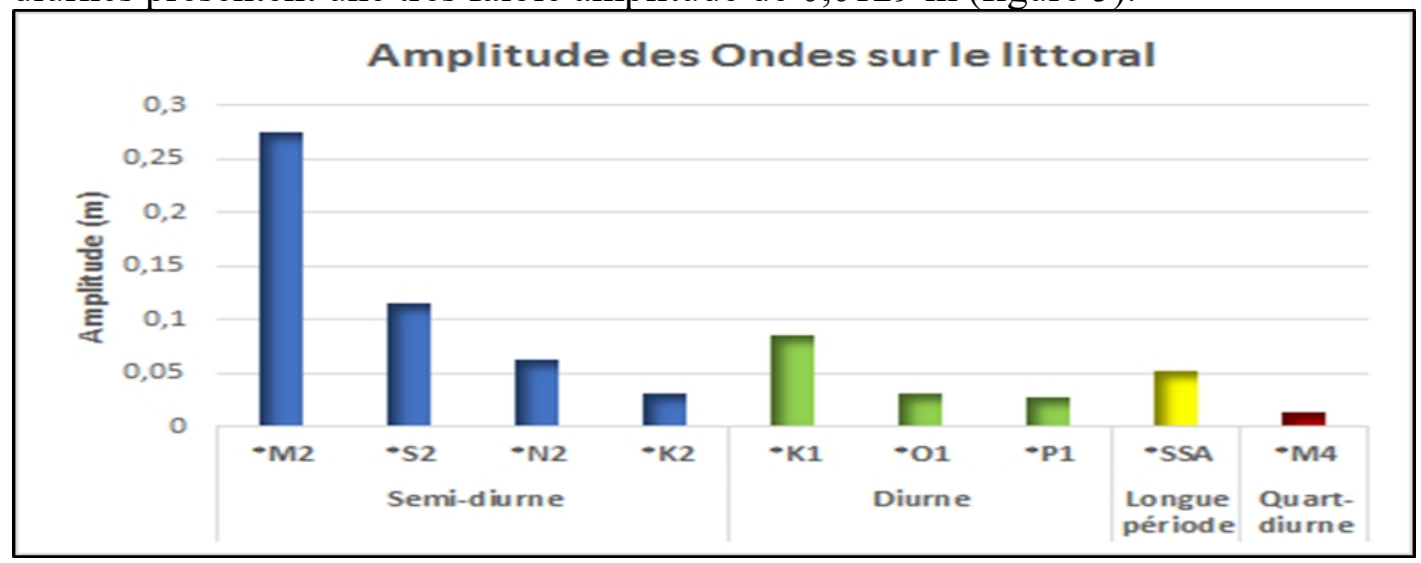

Figure 5 : Amplitude des ondes représentatives sur le littoral Ivoirien

Les phases des ondes diurnes sont largement supérieures à celles des ondes semi-diurnes, avec l'onde K1, indiquant une phase de $354,59^{\circ}$. Tandis que celles des quart-diurnes et longues périodes représentent les plus petites phases soit respectivement $57,08^{\circ}$ et $59,55^{\circ}$ (Tableau I et figure 6).

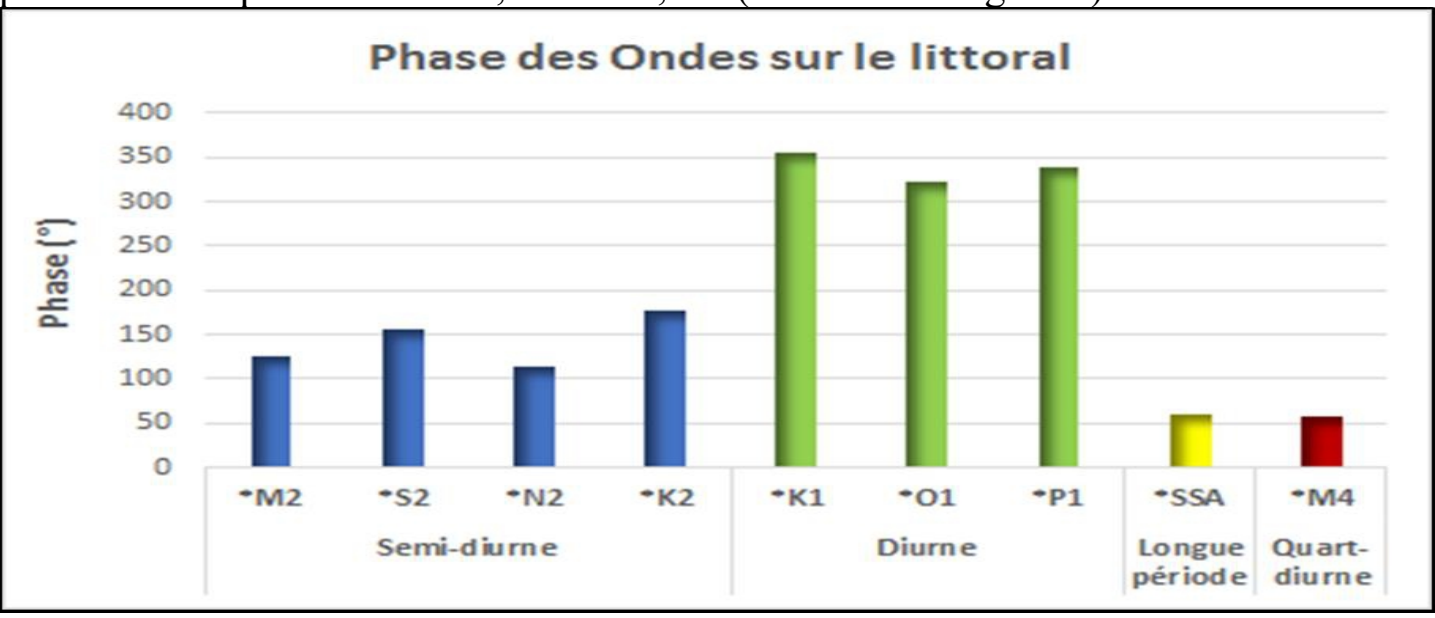

Figure 6 : Phase des ondes représentatives sur le littoral Ivoirien

Ces principales harmoniques permettent de donner les caractéristiques de la marée sur le littoral ivoirien :

- le coefficient de Van der Stock (R) est égal à 0,299. Ce qui signifie qu'en mer la marée est effectivement de type semi-diurne à inégalité diurne,

- le retard moyen de la pleine mer semi-diurne réelle par rapport au passage de la lune dans le plan méridien (Em) en mer est de 4 h 18 min,

- 1'âge de la marée semi-diurne T2 est de 1 jour et 5 h $51 \mathrm{~min}$, ce qui correspond au retard des vives eaux par rapport aux syzygies, 
- 1'ampleur de l'asymétrie tidale générée est de 0,047,

- le sens de l'asymétrie est de $192,84^{\circ}$. Ce qui sous-entend qu'en mer la durée du jusant est plus courte et plus rapide que celle du flot.

\section{Caractéristique des principales composantes harmoniques en lagune Ebrié}

Les composantes semi-diurnes ont également les fréquences les plus élevées en lagune, ensuite les composantes diurnes, puis les longues périodes et enfin les quart-diurnes (tab II).

\begin{tabular}{llll} 
& Tableau II : Principales composantes harmoniques en lagune \\
\hline Onde & Fréquence & Amplitude & Phase \\
\hline$*$ M2 & 0,0805114 & 0,2016 & 158,88 \\
$*$ S2 & 0,0833333 & 0,0659 & 193,87 \\
*N2 & 0,0789992 & 0,0398 & 158,43 \\
K2 & 0,0835615 & 0,0179 & 216,27 \\
*K1 & 0,0417807 & 0,0666 & 18,15 \\
*O1 & 0,0387307 & 0,0156 & 339,09 \\
*P1 & 0,0415526 & 0,0136 & 24 \\
*SSA & 0,0002282 & 0,0965 & 74,42 \\
*M4 & 0,1610228 & 0,0028 & 195,42 \\
\hline
\end{tabular}

L'amplitude de l'onde M2 avec 0,2016 m et une phase de 158,88 est également la plus élevée des ondes semi-diurnes ainsi que de toutes les autres ondes en lagune. Celle de K1 avec 0,0666 m d'amplitude est en tête des ondes diurnes avec une phase de $18,15^{\circ}$. L'amplitude des ondes longues périodes avec $0,0965 \mathrm{~m}$ est plus élevée en lagune et celle des quart-diurne est quasinulle avec $0,0028 \mathrm{~m}$ (Tableau II et figure 7).

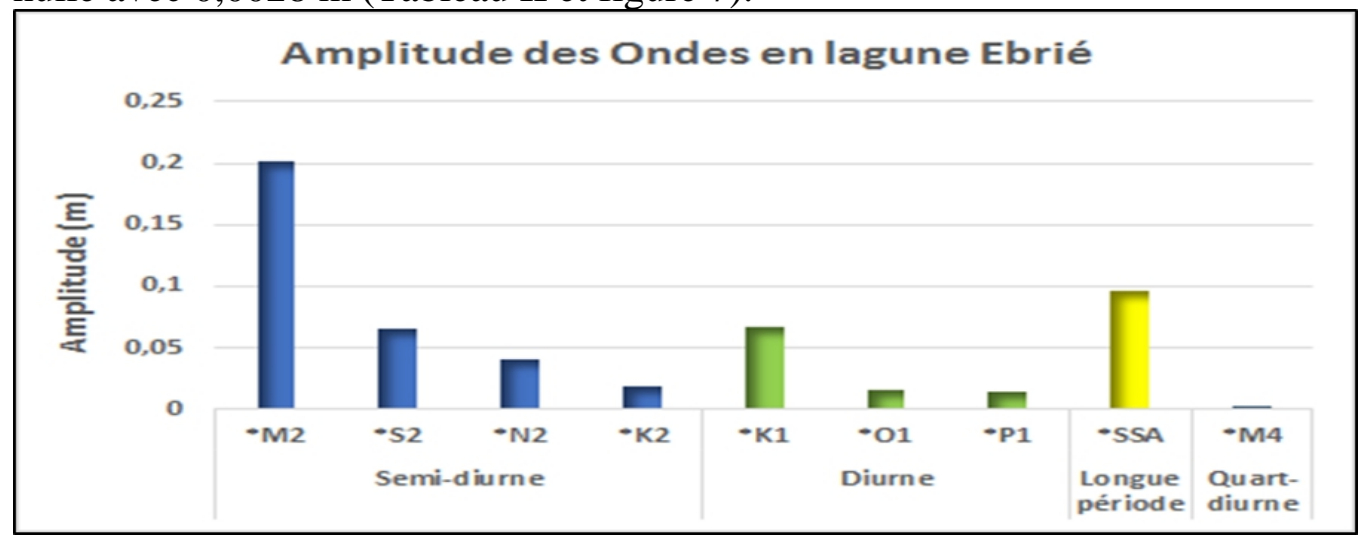

Figure 7 : Amplitude des ondes représentatives en lagune Ebrié.

Seule la phase de l'onde O1 des ondes diurnes avec $339,09^{\circ}$, est supérieure à celles de toutes les autres ondes, tandis que les phases des autres 
ondes diurnes sont moins significatives. Celle des quart-diurnes est devenue importante en lagune soit $195,42^{\circ}$ (Tableau II et fig 8).

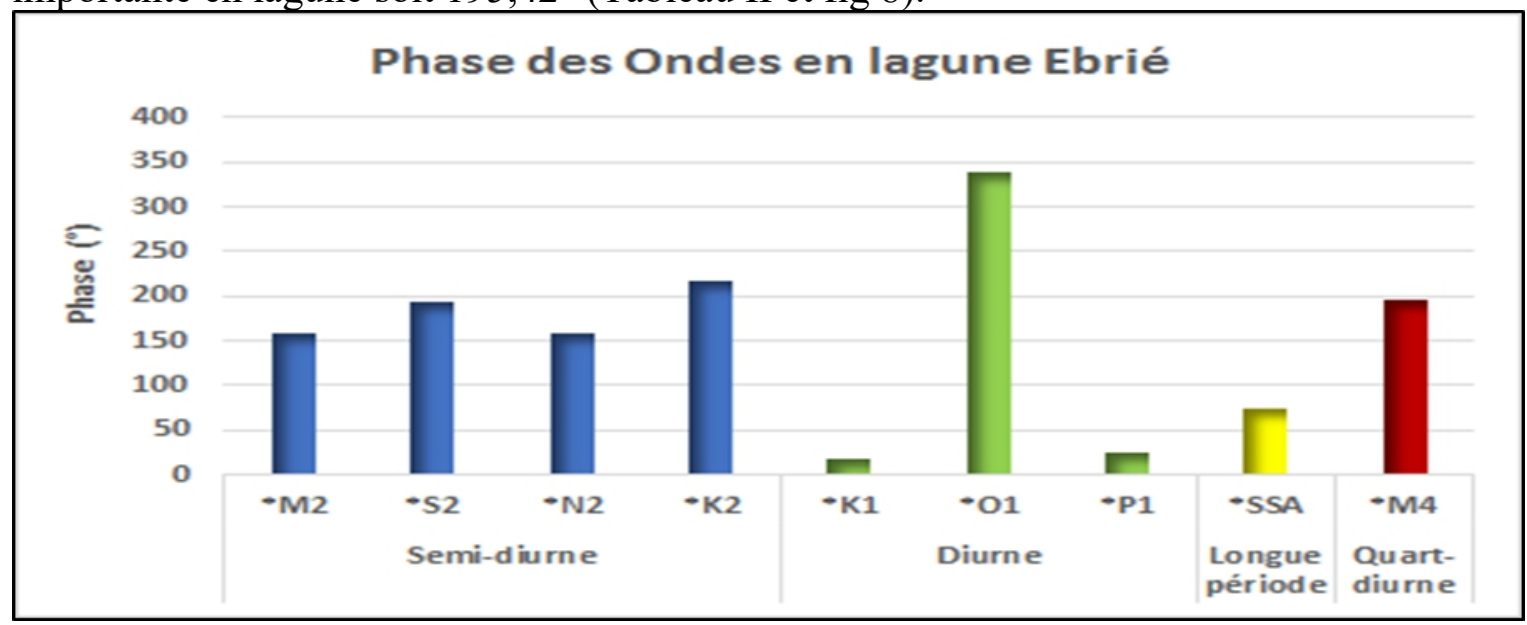

Figure 8: Phase des ondes représentatives en lagune Ebrié.

Ces principales harmoniques ont permis de montrer les caractéristiques de la marée en lagune: le coefficient de Van der Stock (R) est de 0,307. Ce qui implique qu'en lagune la marée est également de type semi-diurne à inégalité diurne. L'établissement moyen est de 5 h 28 min. L'âge de la marée semidiurne T2 est de 1 jour 10 h 26 min. L'ampleur de l'asymétrie tidale générée est de 0,014 , et le sens de l'asymétrie est de $119,34^{\circ}$. Cela montre qu'en lagune la durée du flot est plus courte et rapide que celle du jusant.

\section{Propagation de l'onde de marée dans les différents secteurs de la lagune Ebrié}

La propagation de l'onde de marée du littoral vers la lagune a été étudiée sur les trois différentes parties du système lagunaire Ebrié, plus précisément à partir de l'entrée du Canal de Vridi côté mer (marégraphe I.2).

(a) L'estuaire étant une zone où ont lieu les échanges directs entre la mer et la lagune, plusieurs études y ont été effectuées. La propagation de l'onde de chaque côté de l'estuaire (Est/Ouest) a été étudiée. Cette dernière a également été observée en fonction des différentes saisons (Crue/ étiage).

(a) Pendant l'étiage, différentes vitesses ont été calculées en fonction des bassins (mer, canal et lagune) (Tableau III) :

- entre le littoral et le Canal de Vridi distant de 2,8 km, un temps de propagation de $21 \mathrm{mn} 35 \mathrm{~s}$ avec une vitesse de $2,22 \mathrm{~m} . \mathrm{s}^{-1}$ ont été observés.

- De la mer en lagune sur une distance de $6,95 \mathrm{~km}$, le temps de propagation de l'onde de marée est de $27 \mathrm{mn} 16 \mathrm{~s}$ avec une vitesse de 4,24 m.s 1 . 
Tableau III : Vitesse de propagation de l'onde de marée pendant la saison d'étiage

\begin{tabular}{llll}
\hline Bassins & Distances $(\mathbf{m})$ & Temps de propagation & $\begin{array}{l}\text { Vitesse de } \\
\text { propagatio } \\
\text { n }\end{array}$ \\
\hline Mer-sortie Canal & 2806,69967 & $21 \mathrm{mn} 35 \mathrm{~s}$ & $2,22 \mathrm{~m} / \mathrm{s}$ \\
Mer-Lagune & 6951,709408 & $27 \mathrm{mn} 16 \mathrm{~s}$ & $4,24 \mathrm{~m} / \mathrm{s}$ \\
\hline
\end{tabular}

$\left(a_{2}\right)$ Pendant la saison de crue, les vitesses observées dans les bassins (mer, canal et lagune) (Tableau IV) sont :

- le temps de propagation de l'onde de marée est de $43 \mathrm{mn} 50 \mathrm{~s}$ avec une vitesse de $1,07 \mathrm{~m} . \mathrm{s}^{-1}$ entre la mer et le Canal de Vridi

- De la mer en lagune, le temps de propagation de l'onde de marée est de $19 \mathrm{mn} 1 \mathrm{~s}$ avec une vitesse de $6,09 \mathrm{~m} . \mathrm{s}^{-1}$.

Tableau IV : Vitesse de propagation de l'onde de marée pendant la saison de Crue

\begin{tabular}{llll}
\hline Bassins & Distances $(\mathbf{m})$ & Temps de propagation & $\begin{array}{l}\text { Vitesse de } \\
\text { propagation }\end{array}$ \\
\hline Mer-sortie Canal & 2806,69967 & $43 \mathrm{mn} 50 \mathrm{~s}$ & $1,07 \mathrm{~m} / \mathrm{s}$ \\
Mer-Lagune & 6951,709408 & $19 \mathrm{mn} 1 \mathrm{~s}$ & $6,09 \mathrm{~m} / \mathrm{s}$ \\
\hline
\end{tabular}

Dans l'estuaire Est de la lagune Ebrié c'est-à-dire du côté de GrandBassam, la marée se propage à une vitesse de $6,06 \mathrm{~m} \cdot \mathrm{s}^{-1}$ sur une distance de $18,2 \mathrm{~km}$. Par contre dans la partie Ouest, elle se propage avec une vitesse de $14,33 \mathrm{~m} . \mathrm{s}^{-1}$ sur une distance de $17,2 \mathrm{~km}$ (Tableau V).

Tableau V : Marnage et vitesse de propagation de l'onde de marée dans l'estuaire

\begin{tabular}{|l|l|l|l|l|}
\hline & Distances & Marnage & Heure de la PM & $\begin{array}{l}\text { Vitesse } \\
\text { propagation }\end{array}$ \\
\hline Vridi-Mer (I.2) & $0 \mathrm{~m}$ & $50 \mathrm{~cm}$ & $0 \mathrm{~h} 00 \mathrm{~min}$ & $0 \mathrm{~m} / \mathrm{s}$ \\
\hline Estuaire côté Est (I.4) & $18200 \mathrm{~m}$ & $26 \mathrm{~cm}$ & $0 \mathrm{~h} 50 \mathrm{~min}$ & $6,06 \mathrm{~m} / \mathrm{s}$ \\
\hline Estuaire côté Ouest (I.5) & $17200 \mathrm{~m}$ & $29 \mathrm{~cm}$ & $0 \mathrm{~h} 20 \mathrm{~min}$ & $14,33 \mathrm{~m} / \mathrm{s}$ \\
\hline
\end{tabular}

(b) Le chenal Est de la lagune Ebrié s'étendant d'Abidjan à GrandBassam avec à l'extrémité le fleuve Comoé. La progression de l'onde de marée dans cette partie de la lagune a été calculée dans quatre stations à partir du signal de marée (I.2) en mer et réparties comme suit (Tableau VI) :

- La première, l'Ile des chauves-souris est distant de $26 \mathrm{~km}$ du Canal, la marée se propage dans cette zone avec une vitesse de $6,19 \mathrm{~m} \cdot \mathrm{s}^{-1}$ et un marnage de $19 \mathrm{~cm}$.

- La station de l'Ile Vitré est située à $37 \mathrm{~km}$ du littoral et la marée s'y propage à une vitesse de $5,6 \mathrm{~m} / \mathrm{s}$ avec un marnage de $18 \mathrm{~cm}$.

- Le Bac de Moossou est à $49 \mathrm{~km}$ du canal de Vridi, et la vitesse de propagation dans cette zone est de $5,1 \mathrm{~m} / \mathrm{s}$ avec un marnage de $17 \mathrm{~cm}$.

- La lagune Ouladine se trouvant à $52 \mathrm{~km}$ du canal de Vridi, présente un marnage de $14 \mathrm{~cm}$ et la marée s'y propage avec une vitesse de $4,56 \mathrm{~m} / \mathrm{s}$ 
Tableau VI : Marnage et vitesse de propagation de l'onde de marée de Vridi à Bassam

\begin{tabular}{|l|l|l|l|l|}
\hline & Distances & Marnage & Heure de la PM & $\begin{array}{l}\text { Vitesse de } \\
\text { propagation }\end{array}$ \\
\hline VridiMer (I.2) & $0 \mathrm{~m}$ & $50 \mathrm{~cm}$ & $0 \mathrm{~h} 00 \mathrm{~min}$ & $0 \mathrm{~m} / \mathrm{s}$ \\
\hline Ile des chauves-souris (II.2) & 26000 & 19 & $1 \mathrm{~h} 10$ & $6,19 \mathrm{~m} / \mathrm{s}$ \\
\hline Ile Vitré (II.3) & 37000 & 18 & $1 \mathrm{~h} 50$ & $5,6 \mathrm{~m} / \mathrm{s}$ \\
\hline Moossou (II.4) & 49000 & 17 & $2 \mathrm{~h} 40$ & $5,1 \mathrm{~m} / \mathrm{s}$ \\
\hline Ouladine (II.5) & 52000 & 14 & $3 \mathrm{~h} 10$ & $4,56 \mathrm{~m} / \mathrm{s}$ \\
\hline
\end{tabular}

(c) Le chenal Ouest de la lagune Ebrié concerne la zone d'Abidjan à Asagny. Dans cette partie de la lagune la progression de l'onde de marée a également été suivie à partir de quatre stations de mesure (tableau VII):

- L'Ile cornuet distant de 28,4 km du canal de Vridi, la marée se propage avec une vitesse de $13,52 \mathrm{~m} . \mathrm{s}^{-1}$ avec un marnage de $14 \mathrm{~cm}$.

- L'Ile aux pigeons est située à $48,4 \mathrm{~km}$ du littoral et la marée se propage dans cette zone avec une vitesse de $2,99 \mathrm{~m} / \mathrm{s}$ et un marnage de $4 \mathrm{~cm}$.

- A Potou 31 situé à $64,4 \mathrm{~km}$ du littoral, la vitesse de propagation de l'onde de marée est de $3,97 \mathrm{~m} / \mathrm{s}$ avec un marnage de $5 \mathrm{~cm}$.

- Asagny se trouve à $94,9 \mathrm{~km}$ du littoral, la marée s'y propage avec une vitesse de $5,86 \mathrm{~m} / \mathrm{s}$ avec un marnage de $8 \mathrm{~cm}$.

Tableau VII : Marnage et vitesse de propagation de l'onde de marée de Vridi à Asagny

\begin{tabular}{|l|l|l|l|l|}
\hline & Distances & Marnage & Heure de la PM & Vitesse de propagation \\
\hline Vridi Mer (I.2) & $0 \mathrm{~m}$ & $50 \mathrm{~cm}$ & $0 \mathrm{~h} 00 \mathrm{~min}$ & $0 \mathrm{~m} / \mathrm{s}$ \\
\hline Ile cornuet (III.2) & 28400 & 14 & $0 \mathrm{~h} 35$ & $13,52 \mathrm{~m} / \mathrm{s}$ \\
\hline Ile aux pigeons (III.3) & 48400 & 4 & $4 \mathrm{~h} 30$ & $2,99 \mathrm{~m} / \mathrm{s}$ \\
\hline Potou 31 (III.4) & 64400 & 5 & $4 \mathrm{~h} 30$ & $3,97 \mathrm{~m} / \mathrm{s}$ \\
\hline Asagny (III.5) & 94900 & 8 & $4 \mathrm{~h} 30$ & $5,86 \mathrm{~m} / \mathrm{s}$ \\
\hline
\end{tabular}

\section{INTERPRETATIONS ET DISCUSSIONS}

Niveaux d'eaux et principales composantes harmoniques en mer et en lagune

Le niveau des basses mers est plus faible en mer soit 0,26 $\mathrm{m}$ par rapport aux différents niveaux dans le Canal 0,55 m et en lagune 0,54 m. Le marnage observé en période de vive-eau est 1,2 $\mathrm{m}$ en mer et le niveau moyen durant un cycle annuel est de 1,06 $\mathrm{m}$. Il est quasiment égal à celui en lagune $1,07 \mathrm{~m}$ avec un marnage de $0,8 \mathrm{~m}$. Ce qui n'est le cas dans le Canal de Vridi, où l'on observe un niveau moyen bien élevée soit $1,17 \mathrm{~m}$ avec un marnage de $0,98 \mathrm{~m}$ à cause de sa morphologie restreinte.

Le coefficient de Van der Stock (R) sur le littoral d'Abidjan est de 0,299, en lagune Ebrié 0,307 et sur le littoral de San-Pedro 0,301 (Mahan et $a l$, 2009). Cela montre qu'en Côte d'Ivoire, la marée est effectivement de type semi diurne à inégalité diurne. Le retard moyen de la pleine mer semi-diurne réelle par rapport au passage de la lune dans le plan méridien (Em) en mer est 
de 4 h 18 min et en lagune de 5 h 28 min. Ce qui montre un retard de $1 \mathrm{~h} 10$ min de l'établissement moyen en lagune. L'âge de la marée semi-diurne T2 est de 1jour 5 h 51 min tandis que celle en lagune est de 1 jour 10 h 26 min. Cette différence équivaut à un retard de 4 h 35 min. L'ampleur de l'asymétrie tidale générée en mer étant de 0,047 est plus importante que celle en lagune 0,014. Le sens de l'asymétrie en mer est de $192,84^{\circ}$, tandis que celle en lagune est de $119,34^{\circ}$. Cela sous-entend qu'en mer la durée du flot est plus longue et plus lente que celle du jusant, mais cette tendance de l'asymétrie en mer est inversée en lagune. La durée du flot est plus courte et rapide que celle du jusant.

\section{Vitesse et temps de propagation de l'onde dans les différentes parties de la lagune Ebrié}

L'onde de marée se propage beaucoup plus vite dans la partie Ouest du système lagunaire Ebrié qui est sans influence fluviale. Ainsi sur une distance estimée à $1 \mathrm{~km}$, on détermine un temps de parcours de $20 \mathrm{mn} 48 \mathrm{~s}$ et une vitesse de $0,83 \mathrm{~m} / \mathrm{s}$. Ces résultats sont quasi-identiques à ceux du port de San-Pedro. Pour une distance estimée à 975 m entre le marégraphe du quai de contrainte (port d'office) et le quai du port de pêche, un temps de parcours de $18 \mathrm{mn} 45 \mathrm{~s}$ avec une vitesse de $0,8665 \mathrm{~m} / \mathrm{s}$ est déterminé (Mahan et al, 2009). $\mathrm{Au}$ fur et mesure qu'on s'éloigne du littoral et qu'on se rapproche du canal d'Asagny la marée tend à s'annuler.

Par contre dans la partie Est de la lagune, la marée se propage lentement à cause de l'influence fluviale. L'onde parcours $1 \mathrm{~km}$ en $50 \mathrm{mn} 30$ $\mathrm{s}$ avec une vitesse de $0,33 \mathrm{~m} / \mathrm{s}$.

Dans le Canal de Vridi, l'onde se propage assez rapidement pendant la saison sèche, elle parcourt le canal en $21 \mathrm{mn} 05 \mathrm{~s}$ avec une vitesse $0,79 \mathrm{~m} / \mathrm{s}$, par contre en saison de crue est beaucoup plus ralentit, on a deux fois le temps de parcours en saison sèche soit $43 \mathrm{~min} 51 \mathrm{~s}$ avec une vitesse de $0,38 \mathrm{~m} / \mathrm{s}$. Ces résultats sont en accord avec ceux de Varlet (1978) dans son ouvrage intitulé le "régime de la lagune Ebrié".

On remarque ainsi que quel que soit la saison, la marée se propage plus vite du côté Ouest de la lagune que dans le Canal à cause de la morphologie restreinte du Canal de Vridi (Samassy et al, 2017) et de la faible profondeur de la lagune car selon Simon (2007), la marée se propage plus vite sur de petits fonds. Et dans la partie est de la lagune Ebrié, au fur et à mesure qu'on se rapproche du fleuve Comoé, la vitesse de propagation de l'onde de marée diminue. En période de d'étiage, l'influence marine est dominante et les plus hauts niveaux d'eau sont observés en mer. Par contre, en période de crue, les plus hauts niveaux d'eaux sont observés en lagune (figure 9). 


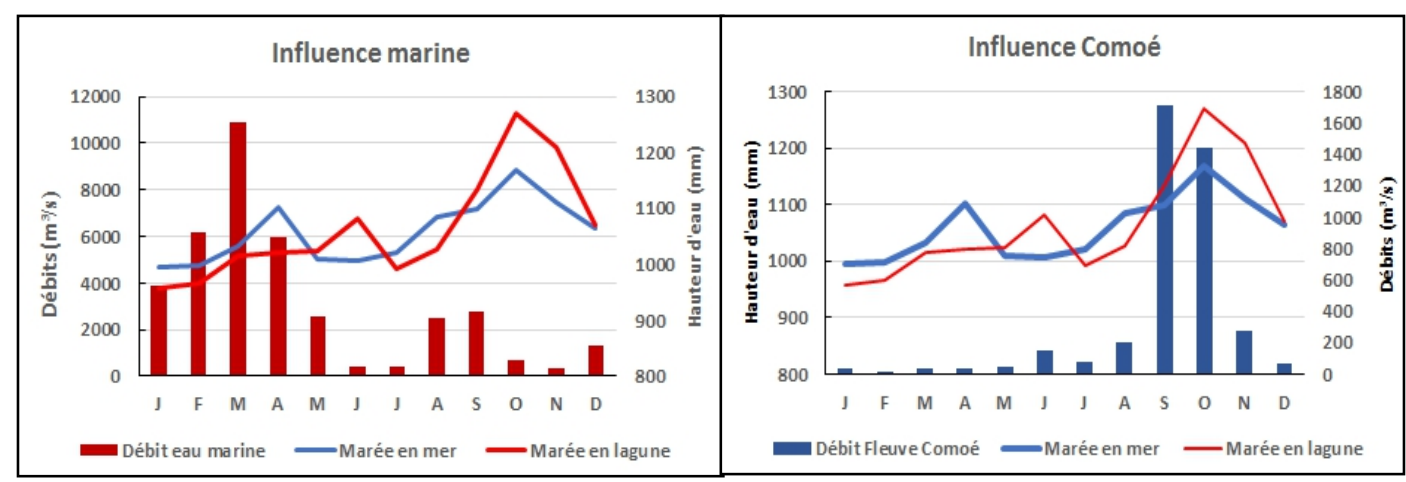

Figure 9 : Influence marine et fluviale sur le signal de marée en lagune Ebrié

\section{CONCLUSION}

En définitive, on retient que lors de la propagation du signal de marée en lagune Ebrié, la pleine mer arrive du côté Ouest de la lagune en 57 s plutôt que dans le canal de Vridi pendant la saison sèche et $23 \mathrm{~min}$ après pendant la saison de crue du fleuve Comoé. Dans la partie Est, ce n'est que 30 min après la pleine mer de la partie Ouest que le maximum est atteint.

L'onde de marée se propage beaucoup plus vite en lagune que dans le Canal de Vridi à cause de la faible profondeur de la lagune. Mais elle est retardée par la crue du fleuve Comoé. En définitive on peut dire que la propagation du signal de marée en lagune Ebrié serait dépendante de la morphologie des bassins et des apports fluviaux.

La partie ouest du système lagunaire Ebrié serait ainsi une zone favorable à la propagation du signal de marée, par contre la partie Est, serait une zone très influencée par les apports fluviaux entravant ainsi la propagation de l'onde marée.

\section{REMERCIEMENTS}

Les auteurs de cet article remercient la direction des infrastructures du Port Autonome d'Abidjan pour la mise à disposition des archives des services hydrographie et océanographie. Nous remercions également le service hydrographie et océanographie de la marine française (SHOM) pour les données marégraphiques historiques du littoral ivoirien; ce qui a favorisé l'amélioration de cet article. Et tous ceux qui ont contribués de près et de loin à la réalisation de ce travail scientifique.

\section{References:}

1. Dufour, P. (1982). Les frontières naturelles et humaines du système lagunaire Ebrié.

2. Incidences sur l'hydroclimat, Hydrobiologia 94, pp. 105-120. 
3. Gouriou, T. (2012). Evolution des composantes du niveau marin à partir d'observations de marégraphie effectuées depuis la fin du 18 ème siècle en Charente-Maritime. Sciences de la Terre. Thèse de Doctorat, Université de La Rochelle, 475 pp.

4. Mahan, C. N., J. Abe, A. Angora et S. BAMBA. (2009). Propagation of tidal waves in the san-pedro harborwest coast of Cote d'Ivoire rev. ivoir. Sci. Technologies $13: 77$ - 88 in 1813-3290.

5. Monde, S. (2004). Etude et modélisation hydrodynamique de la circulation des masses d'eau dans la lagune Ebrié (Côte d'Ivoire). Thèse de Doctorat d'Etat, Univ Abidjan, $324 \mathrm{p}$.

6. Ramany, B.P. (1979). Le système lagunaire Ebrié en Côte d'Ivoire : fonctionnement hydrodynamique et salinité. Thèse de Doctorat d'Ingénieur. Institut National Polytechnique de Toulouse, $179 \mathrm{p}$.

7. Samassy, R., K. B. Djè, E. W. Yéo, S. Sangaré et S. Monde. (2017). Incidences des forçages naturels dans la variabilité saisonnière du signal marégraphique du littoral d'Abidjan (Côte d'Ivoire), Bioterre Volume $17: 62-76$

8. Simon, B. (2007). La marée océanique cotière, Paris: Institut océanographique, $433 \mathrm{p}$.

9. Varlet, F. (1978). Le régime de la lagune Ebrié (Côte d'Ivoire). Trav. Doc. Orstom, $83 \mathrm{p}$.

10. Wango, T. (2009). Modélisation hydrodynamique de la dispersion du sel et de l'eau douce dans le complexe lagunaire de la Côte d'Ivoire. Thèse de Doctorat, Université Houphouët Boigny, $\mathrm{n}^{0} 179$, 165p. 